\title{
A VAN KAMPEN THEOREM FOR SEPARABLE ALGEBRAS
}

\author{
ANDY R. MAGID
}

ABstract. If the commutative ring $S$ is the fibre product of $R_{1}$ and $R_{2}$ over $R$, and if $R_{2}$ maps onto $R$, then the fundamental group of $S$ is the profinite free product of the fundamental groups of $R_{1}$ and $R_{2}$ with the fundamental group of $R$ amalgamated.

Van Kampen's theorem in algebraic topology asserts that, under suitable hypotheses, if

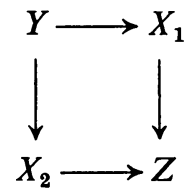

is a co-cartesian square of topological spaces, the corresponding diagram

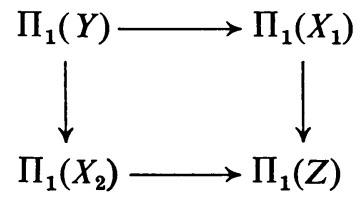

of fundamental groups is also co-cartesian, i.e., that $\Pi_{1}(Z)$ is the free product of $\Pi_{1}\left(X_{1}\right)$ and $\Pi_{1}\left(X_{2}\right)$ with the image of $\Pi_{1}(Y)$ amalgamated. van Kampen's proof is in [9]; an elementary exposition may be found in [5, Chapter II]. The present paper contains a proof of the analogue of van Kampen's theorem for fundamental groups of commutative rings. (By the fundamental group of a commutative ring we mean the profinite fundamental group in the sense of Grothendieck [8] of the corresponding affine scheme; this is the same as the Galois group of the separable closure of the ring.) As will be seen below, the theorem is a simple consequence of results of Milnor and Bass.

All the rings considered here are commutative with 1 , and $\Pi(R)$ denotes the fundamental group of the commutative ring $R$ (for $\Pi(R)$ to be defined $R$ must have no idempotents except zero and one). If $G_{1}$ and $G_{2}$ are

Received by the editors September 8, 1972.

AMS (MOS) subject classifications (1970). Primary 13B05; Secondary 14E20.

Key words and phrases. Separable algebra, profinite group, fibre product, cartesian square, co-cartesian square, fundamental group. 
profinite groups, $\operatorname{Hom}_{c}\left(G_{1}, G_{2}\right)$ denotes the continuous homomorphisms from $G_{1}$ to $G_{2}$, and $\mathscr{M}\left(G_{1}\right)$ denotes the category of finite sets on which $G_{1}$ acts continuously on the left with morphisms being $G_{1}$-equivariant maps.

Definition 1. Suppose that in $\left(G, G_{1}, G_{2}, f_{1}, f_{2}\right), G, G_{1}, G_{2}$ are profinite groups and $f_{i}: G \rightarrow G_{i}$ are continuous maps. A profinite free product with amalgamation of this five-tuple is a profinite group $E$ and continuous maps $j_{i}: G_{i} \rightarrow E$ such that $j_{1} f_{1}=j_{2} f_{2}$ and for any finite $H$ the map

$$
\operatorname{Hom}_{c}(E, H) \rightarrow \operatorname{Hom}_{c}\left(G_{1}, H\right) \times \operatorname{Hom}_{c}(G, H) \operatorname{Hom}_{c}\left(G_{2}, H\right)
$$

given by sending $h$ to $\left(h j_{1}, h j_{2}\right)$ is a bijection. We denote $E$ by $G_{1} \hat{*}_{G} G_{2}$ and, for $g_{i} \in G_{i}, j_{1}\left(g_{1}\right) j_{2}\left(g_{2}\right)$ by $g_{1} * g_{2}$.

Profinite free products exist; for lack of an available reference we sketch a proof here.

Proposition 2. Let $\left(G, G_{1}, G_{2}, f_{1}, f_{2}\right)$ be as in Definition 1. Then a profinite free product with amalgamation of this five-tuple exists. The elements of the form $a_{1} * b_{1} * a_{2} * b_{2} * \cdots * a_{n} * b_{n}$ (where $a_{i}$ is in $G_{1}$, $b_{i}$ is in $G_{2}$ and $n$ ranges over the positive integers) are dense in the free product.

Proof. We denote the ordinary free product with amalgamation of $\left(G, G_{1}, G_{2}, f_{1}, f_{2}\right)$ by $G_{1} *_{G} G_{2}$ (see [6, Chapter 17] for the theory of such products). Let $h_{i}: G_{i} \rightarrow H$ be a pair of continuous homomorphisms with range the finite group $H$ such that $h_{1} f_{1}=h_{2} f_{2}$, and let $N\left(h_{1}, h_{2}\right)$ be the kernel of the homomorphism $G_{1} *_{G} G_{2} \rightarrow H$ induced by the $h_{i}$. Since we have (in the obvious notation) $N\left(h_{1}, h_{2}\right) \cap N\left(k_{1}, k_{2}\right)=N\left(h_{1} \times k_{1}, h_{2} \times k_{2}\right)$ the set $\mathscr{N}$ of all such subgroups is a basis at the identity for a topology on $G_{1} *_{G} G_{2}$ and in this topology the homomorphisms $G_{i} \rightarrow G_{1} *_{G} G_{2}$ are continuous. $\mathscr{N}$ is directed by inverse inclusion and we let $G_{1} \hat{*}_{G} G_{2}$ be the inverse limit of $G_{1}{ }^{*} G G_{2} / N$ as $N$ ranges over $\mathscr{N}$ and $j_{i}: G_{i} \rightarrow G_{1} \hat{*}_{G} G_{2}$ the composite of $G_{i} \rightarrow G_{1} *_{G} G_{2}$ followed by the projection to the inverse limit. We omit the easy verification that this is indeed a profinite free product with amalgamation. The second assertion of the theorem follows from the fact that the projection $G_{1} *_{G} G_{2} \rightarrow G_{1} *_{G} G_{2} / N$ is onto at each stage of the inverse system.

Let $C, C_{1}, C_{2}$ be categories and $F_{i}: C_{i} \rightarrow C$ be functors. Following Bass $\left[1,3.1\right.$, p. 358] we define the fibre product category $C_{1} \times{ }_{C} C_{2}$ to be the category whose objects are triples $\left(X_{1}, a, X_{2}\right)$ where $X_{i}$ is an object of $C_{i}$ and $a: F_{1} X_{1} \rightarrow F_{2} X_{2}$ is an isomorphism, and whose morphisms are pairs $\left(h_{1}, h_{2}\right):\left(X_{1}, a, X_{2}\right) \rightarrow\left(Y_{1}, b, Y_{2}\right)$ where $f_{i}: X_{i} \rightarrow Y_{i}$ is a morphism in $C_{i}$ and $F_{2} h_{2} a=b F_{1} h_{1}$. 
Proposition 3. Let $\left(G, G_{1}, G_{2}, f_{1} f_{2}\right)$ be as in Definition 1. Then $\mathscr{M}\left(G_{1} \hat{*}_{G} G_{2}\right)$ is equivalent to $\mathscr{M}\left(G_{1}\right) \times \mathscr{M}(G) \mathscr{M}\left(G_{2}\right)$. (Here the functor from $\mathscr{M}\left(G_{i}\right)$ to $\mathscr{M}(G)$ is "restriction of operators".)

Proof. An object of the fibre product category is a triple $\left(X_{1}, a, X_{2}\right)$ where $X_{i}$ is a $G_{i}$-set, $a: X_{1} \rightarrow X_{2}$ is a bijection and, for $g \in G$ and $x \in X_{1}$, $a\left(f_{1}(g) x\right)=f_{2}(g)(a x)$. Let $A\left(X_{i}\right)$ be the symmetric group in the set $X_{i}$ and let $g_{i}: G_{i} \rightarrow A\left(X_{i}\right)$ give the $G_{i}$-structure on $X_{i}$. Define $g_{1}^{\prime}: G_{1} \rightarrow A\left(X_{2}\right)$ by $g_{1}^{\prime}(g)=a g_{1}(g) a^{-1}$ for $g \in G_{1}$. Then $g_{1}^{\prime} f_{1}=g_{2} f_{2}$ and since there is an induced continuous map $G_{1 * G} G_{2} \rightarrow A\left(X_{2}\right)$ which makes $X_{2}$ into a $G_{1} \hat{*}_{G} G_{2}$-set which we call $X\left(X_{1}, a, X_{2}\right)$. If $c_{i} \in G_{1}, d_{i} \in G_{2}, i=1,2, \cdots, n$ and $x \in$ $X\left(X_{1}, a, X_{2}\right)$, then $\left(c_{1} * d_{1} * \cdots * c_{n} * d_{n}\right) x=a c_{1} a^{-1} d_{1} \cdots a c_{n} a^{-1} d_{n} x$. Let $\left(h_{1}, h_{2}\right):\left(X_{1}, a, X_{2}\right) \rightarrow\left(Y_{1}, b, Y_{2}\right)$ be a morphism in the fibre product category so $h_{i}: X_{i} \rightarrow Y_{i}$ is a $G_{i}$ map such that, as functions, $h_{2} a=b h_{1}$ (note then that given $a, b$ and $h_{2}, h_{1}$ (if it exists) is unique since $h_{1}=b^{-1} h_{2} a$ ). Let $X\left(h_{1}, h_{2}\right): X\left(X_{1}, a, X_{2}\right) \rightarrow X\left(Y_{1}, a, Y_{2}\right)$ be the function $h_{2}: X_{2} \rightarrow Y_{2}$. We claim that $X\left(h_{1}, h_{2}\right)$ is a morphism of $G_{1} \hat{*}_{G} G_{2}$-sets. Clearly it suffices to check this in a dense subset of the free product, i.e., by Proposition 2, for the elements $c_{1} * d_{1} * \cdots * c_{n} * d_{n}$ with $c_{i} \in G_{1}$ and $d_{i} \in G_{2}$. To simplify notation we take $n=1$. Then, for $x$ in $X_{2}, c$ in $G_{1}$ and $d$ in $G_{2}$,

$$
X\left(h_{1}, h_{2}\right)(c * d)(x)=h_{2} a c a^{-1} d x=b c b^{-1} d h_{2} x=(c * d) X\left(h_{1}, h_{2}\right)(x) .
$$

We note that $X(\cdot)$ is a functor from the fibre product category to $\mathscr{M}\left(G_{1} \hat{*}_{G} G_{2}\right)$ which we claim is an equivalence. The functor is clearly faithful (see the note above). To see it is full, let $h: X\left(X_{1}, a, X_{2}\right) \rightarrow$ $X\left(Y_{1}, b, Y_{2}\right)$ be a $G_{1} \hat{*}_{G} G_{2}$-map. Let $h_{2}: X_{2} \rightarrow Y_{2}$ be $h$ (as a function) and let $h_{1}=b^{-1} h_{2} a$. Let $c \in G_{1}$ and $d \in G_{2}$. Then it is easy to check from the equations $h\left(g_{1} * 1\right)(a x)=\left(g_{1} * 1\right) h(a x) \quad$ (for $\left.x \in X_{1}\right)$ and $h\left(1 * g_{2}\right)(x)=$ $\left(1 * g_{2}\right) h(x)$ (for $\left.x \in X_{2}\right)$ that $h_{i}$ is $G_{i}$-equivalent. By definition $h_{2} a=b h_{1}$ so $\left(h_{1}, h_{2}\right)$ is a morphism in the fibre product category and $X\left(h_{1}, h_{2}\right)=h$. Thus $X(\cdot)$ is full. Finally, to complete the proof that $X(\cdot)$ is an equivalence, we verify that every isomorphism class in $\mathscr{M}\left(G_{1} \hat{*}_{G} G_{2}\right)$ contains an image of $X(\cdot)$. Let $X$ be any object of $\mathscr{M}\left(G_{1} \hat{*}_{G} G_{2}\right)$. Make $X$ a $G_{i}$-set via $g_{i} x=$ $j_{i}\left(g_{i}\right) x$ for $g_{i} \in G_{i}, x \in X$, and call this $G_{i}$-set $X_{i}$. Let $a: X_{1} \rightarrow X_{2}$ be the identity. Then $\left(X_{1}, a, X_{2}\right)$ belongs to the fibre product category and the identity function provides a $G_{1} \hat{*}_{G} G_{2}$-isomorphism $X\left(X_{1}, a, X_{2}\right) \rightarrow X$.

We now turn our attention to separable algebras. We begin by recalling Milnor's theorem ([7, 2.1-2.3, p. 20] and [1, Theorem 5.1, p. 497]): if

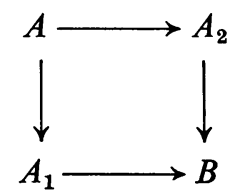


is a cartesian square of commutative rings with $A_{2} \rightarrow B$ surjective, then $P(A)$ is equivalent to $P\left(A_{1}\right) \times_{P(B)} P\left(A_{2}\right)$ (where $P(R)$ is the category of finitely generated projective modules over the ring $R$ ); the equivalence sends an object $M$ of $P(A)$ to $\left(A_{1} \otimes_{A} M, a, A_{2} \otimes_{A} M\right)$ where

$$
a: B \otimes_{A_{1}}\left(A_{1} \otimes_{A} M\right) \rightarrow B \otimes_{A_{2}}\left(A_{2} \otimes_{A} M\right)
$$

is the canonical isomorphism and the inverse functor to the equivalence sends $\left(M_{1}, a, M_{2}\right)$ to $\left\{(x, y)\right.$ in $\left.M_{1} \times M_{2}: a(1 \otimes x)=1 \otimes y\right\}$.

As Bass remarks [1, (5.2), p. 481], Milnor's theorem also applies to various categories of projective modules with structure; one need only check that the equivalence and its inverse functor take values in the subcategory of the modules carrying the structure in question. We apply this to the category of commutative separable algebras whose underlying module is finitely generated and projective (over the ring $R$ this category will be denoted $S(R)$ ).

Proposition 4. Under the hypotheses of Milnor's theorem, $S(A)$ is equivalent to $S\left(A_{1}\right) \times_{S(B)} S\left(A_{2}\right)$.

Proof. If $T$ is an object in $S(A)$, then $A_{i} \otimes_{A} T$ is an object in $S\left(A_{i}\right)$, and it follows that the equivalence of Milnor's theorem takes values in the fibre product category. Now let $\left(T_{1}, a, T_{2}\right)$ be an object of the fibre product category. We need to verify that $M\left(T_{1}, a, T_{2}\right)=M$ (which is clearly an $A$ algebra and by Milnor's theorem a finitely generated projective $A$-module) is a separable $A$-algebra. Since it is finitely generated and projective as an $A$-module, $M$ will be separable if for each maximal ideal $p$ of $A, M / p M$ is a separable $A / p A$-algebra [4, Theorem 7.1(c), p. 72]. But by [1, Proposition 5.11, p. 486], there is a prime ideal $q$ in either $A_{1}$ or $A_{2}$ (say $A_{1}$ ) with $q \cap A=p$. By [7, Theorem 2.3, p. 20], $A_{1} \otimes_{A} M=T_{1}$, so $M / p M \otimes_{A / p A}$ $A_{1} / q A_{1}=T_{1} / q T_{1}$ is $A_{1} / q A_{1}$-separable. Since $A_{1} / q A_{1}$ contains $A / p A$ as $A / p A$-summand, $M / p M$ is $A / p A$-separable [4, Corollary 1.10, p. 45].

Childs gives the analogue of Proposition 4 for Azumaya algebras in [3, §IV]. We now proceed to the main result of this paper.

THEOREM 5. Let

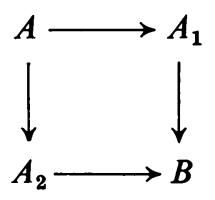

be a cartesian square of commutative rings with no nontrivial idempotents with $A_{1} \rightarrow B$ surjective. Then $\Pi(A)=\Pi\left(A_{1}\right) \hat{*}_{\Pi(B)} \Pi\left(A_{2}\right)$. 
Proof. We begin by remarking that the dual of a fibre product category is the fibre product of the duals. The categories $\mathscr{M}(\Pi(A)), \mathscr{M}\left(\Pi\left(A_{1}\right)\right)$, $\mathscr{M}\left(\Pi\left(A_{2}\right)\right)$ and $\mathscr{M}(\Pi(B))$ are the dual categories to $S(A), S\left(A_{1}\right), S\left(A_{2}\right)$ and $S(B)$ [8, Chapter IV]. Thus by Proposition 4 and the above remark, $\mathscr{H}(\Pi(A))$ is equivalent to $\mathscr{M}\left(\pi\left(A_{1}\right)\right) \times \mathscr{M}(\Pi(B)) \mathscr{M}\left(\Pi\left(A_{2}\right)\right)$. By Proposition 3 this latter category is equivalent to $\left(\Pi\left(A_{1}\right) \hat{*}_{\Pi(B)} \Pi\left(A_{2}\right)\right)$. By $[8,4.4 .1 .11$, p. 87] it follows that $\Pi(A)$ and $\Pi\left(A_{1}\right) \hat{*}_{\Pi(B)} \Pi\left(A_{2}\right)$ are isomorphic since the categories of finite sets on which they act continuously are equivalent.

A list of common situations in which the hypotheses of Theorem 5 are satisfied is found in [1, p. 483]. We consider some representative examples here.

EXAMPLE A. Let $G$ be a cyclic group of prime order $p$ and $Z$ a primitive pth-root of unity. Then [7, p. 29]

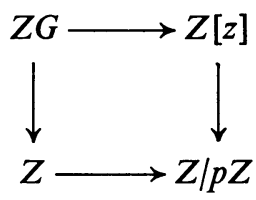

where the left vertical map is augmentation, the right vertical sends $z$ to 1 , the top map sends a generator of $G$ to $z$ and the bottom map is the canonical one is cartesian. Theorem 5 applies and we conclude, using the fact that $\Pi(Z)$ is trivial, that $\Pi(Z[z]) \rightarrow \Pi(Z G)$ is surjective with kernel the image of $\Pi(Z / p Z)$.

EXAMPLE B. Let the compact connected Hausdorff space $X$ be the union of the two connected closed subsets $F_{1}$ and $F_{2}$ and suppose $Y=$ $F_{1} \cap F_{2}$ is also connected. Let $C(\cdot)$ denote the ring of continuous complex valued functions on $(\cdot)$. Then

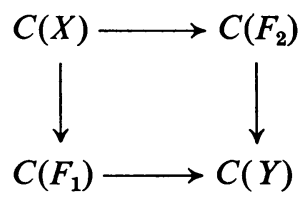

is cartesian. By a result of Childs [2, Theorem 2, p. 30], $\Pi(C(X))$ is the profinite completion of $\Pi_{1}(X)$, and similarly for $F_{1}, F_{2}$ and $Y$. Thus from Theorem 5 we recover a topological van Kampen theorem, at least up to profinite completion.

Example C. Let $R$ be the real numbers, $C$ the complexes, and $A$ the ring of polynomials $f$ over $R$ in the variable $X$ such that $f(\sqrt{ }-1)$ is real. 
Then

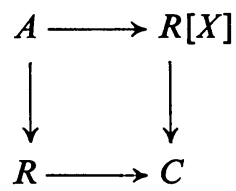

where the horizontal maps are the inclusions, the left map and the right evaluation at $\sqrt{ }-1$, satisfies the hypotheses of Theorem 5 . Of course, $\Pi(C)$ is trivial and $\Pi(R)=Z / 2 Z$. Also $\Pi(R[X])=Z / 2 Z$ (since $C[X]$ is separably closed and Galois over $R[X]$ with group $Z / 2 Z$ ) and hence it follows that $\Pi(A)=Z / 2 Z \hat{*} Z / 2 Z$ (we note that this latter group is nonabelian and infinite since it has every dihedral group as a homomorphic image).

\section{REFERENCES}

1. H. Bass, Algebraic K-theory, Benjamin, New York, 1968. MR 40 \#2736.

2. L. Childs, On covering spaces and Galois extensions, Pacific J. Math. 37 (1971), 29-33.

3. - Mayer-Vietoris sequences and Brauer groups of nonnormal domains, J. Algebra (to appear).

4. F. DeMeyer and I. Ingraham, Separable algebras over commutative rings, Lecture Notes in Math, vol. 181, Springer-Verlag, New York and Berlin, 1971. MR 43 \#6199.

5. A. Gramain, Topologie des surfaces, Presses universitaires de France, Paris, 1971.

6. M. Hall, The theory of groups, Macmillan, New York, 1959. MR 21 \#1996.

7. J. Milnor, Introduction to algebraic K-theory, Ann. of Math. Studies 72, Princeton Univ. Press, Princeton, N.J., 1971.

8. J. Murre, Lectures on an introduction to Grothendieck's theory of the fundamental group, Tata Institute of Fundamental Research, Bombay, 1967.

9. E. van Kampen, On the connection between the fundamental groups of some related spaces, Amer. J. Math. 55 (1933), 261-267.

Department of Mathematics, University of Oklahoma, Norman, Oklahoma 73069 